\title{
AGANGLIONOSE TOTAL dO CÓLON: ASPECTOS RADIOLÓGICOS*
}

\author{
Nelson Marcio Gomes Caserta ${ }^{1}$, Elisa Maria de Brito Pacheco ${ }^{1}$, Mirian Jane Gabriel da Silva ${ }^{2}$, \\ Shoji Miyabara ${ }^{3}$, Rubens Marcondes Pereira ${ }^{4}$
}

\begin{abstract}
Resumo A partir da observação de três pacientes com aganglionose total do cólon e de revisão da literatura, os autores salientam as particularidades diagnósticas desta entidade que justificam uma análise separada da forma típica da doença de Hirschsprung. Não há sinais radiológicos patognomônicos e o diagnóstico definitivo somente pode ser obtido pelo estudo histopatológico. Considerando sua alta incidência de complicações e mortalidade, a aganglionose total do cólon deve ser considerada em todo recém-nascido ou criança maior com evidências radiológicas de obstrução intestinal, quaisquer que sejam os achados de enema opaco. Unitermos: Aganglionose; Doença de Hirschsprung; Obstrução intestinal.
\end{abstract}

\section{Abstract Total aganglionosis of the colon: radiological findings}

The authors present the cases of three patients with total aganglionosis of the colon and review the literature. The main diagnostic features are reviewed to justify a separate analysis of the typical form of Hirschsprung's disease. There are no pathognomonic radiological findings and definitive diagnosis can only be established by histological examination. Considering the high incidence of complications and the mortality of patients with total aganglionosis of the colon, this disease should be suspected in any infant or young child with evidence of bowel obstruction seen on plain films, regardless of the contrast enema findings. Key words: Aganglionosis; Hirschsprung's disease; Intestinal obstruction.

\section{INTRODUÇÃO}

A aganglionose total do cólon (ATC), com ou sem acometimento do intestino delgado, é uma forma da doença de Hirschsprung, que embora rara, tem sido diagnosticada com frequiência crescente ${ }^{(\mathbf{1 , 2})}$. Em estudo comparado, a ATC tem uma incidência maior de complicações, além de certas particularidades diagnósticas e terapêuticas que justificam sua análise separada da forma clássica segmentar da doença de Hirschsprung ${ }^{(3)}$.

Considerando-se que não há aspectos clínicos, radiológicos ou manométricos específicos para a ATC, o objetivo desta apresentação é alertar pediatras, radiologistas e cirurgiões pediátricos para a inclusão desta possibilidade diagnóstica diante de toda criança com evidências de obstrução intestinal.

\footnotetext{
* Trabalho realizado no Hospital das Clínicas da Faculdade de Ciências Médicas da Universidade Estadual de Campinas (FCM-Unicamp), Campinas, SP.

1. Professor Doutor de Radiologia.

2. Médica Radiologista da Casa de Saúde de Campinas.

3. Professor Assistente da Disciplina de Cirurgia Pediátrica.

4. Professor Doutor de Radiologia, Hospital Vera Cruz Campinas.

Endereço para correspondência: Prof. Dr. Nelson M.G. Caserta. Rua Barão de Atibaia, 338. Campinas, SP, 13023-010. E-mail: ncaser@mpcnet.com.br

Recebido para publicação em 19/3/2003. Aceito, após revisão, em 10/9/2003.
}

\section{MATERIAIS E MÉTODOS}

Foram revistos os dados clínicos, as radiografias simples e os exames contrastados do cólon de três pacientes com o diagnóstico pós-operatório confirmado de ATC. Todas as crianças apresentavam constipação crônica desde o nascimento, foram atendidas com o diagnóstico clínico de obstrução intestinal e tiveram o diagnóstico pós-operatório de ATC, sem acometimento do intestino delgado.

Os dados dos exames de imagem foram comparados com aqueles referidos na literatura a respeito das diversas formas de apresentação radiográfica da ATC.

\section{RESULTADOS}

O caso 1 é de paciente do sexo feminino, de 45 dias de idade, desnutrida, com distensão abdominal e vômitos. A radiografia simples (Figura 1A) mostrava distensão intestinal, sem sinais de ar na ampola retal. O enema opaco mostrava cólon curto, de calibre normal (Figura 1B). A radiografia, 24 horas após o enema opaco, revelava a persistência do material de contraste em praticamente todo o cólon (Figura 1C) e a presença de espasmos no sigmóide.

$\mathrm{O}$ caso 2 refere-se a paciente do sexo masculino, de dois anos de idade, cuja ra- diografia simples (Figura 2A) revelava abundante conteúdo em alças distendidas. $\mathrm{O}$ enema opaco mostrava discreta distensão do hemicólon direito e o cólon transverso contendo grande quantidade de material fecal retido (Figura 2B).

$\mathrm{O}$ caso 3 diz respeito a paciente do sexo masculino, de 12 anos de idade. A radiografia simples (Figura 3A) mostrava acentuada distensão de alças intestinais e imagens de resíduos fecais em grande quantidade. O enema opaco (Figura 3B) revelava segmentos cólicos distendidos, com abundante retenção de resíduos fecais.

Todos os pacientes foram submetidos a laparotomia e ileostomia, com biópsia dos segmentos colônicos e do íleo distal, que demonstraram as alças colônicas aganglionares. Cirurgia de abaixamento foi realizada em segundo tempo.

\section{DISCUSSÃO}

A doença de Hirschsprung geralmente acomete um segmento aganglionar curto do cólon distal, limitando-se ao reto e sigmóide em $70 \%$ dos casos. Entretanto, comprometimento mais extenso de todo o cólon e intestino delgado pode ocorrer em $1 \%$ a $10 \%$ dos casos, podendo atingir até o duodeno ${ }^{(4-6)}$. Esta forma total de aganglionose intestinal seria herdada como 


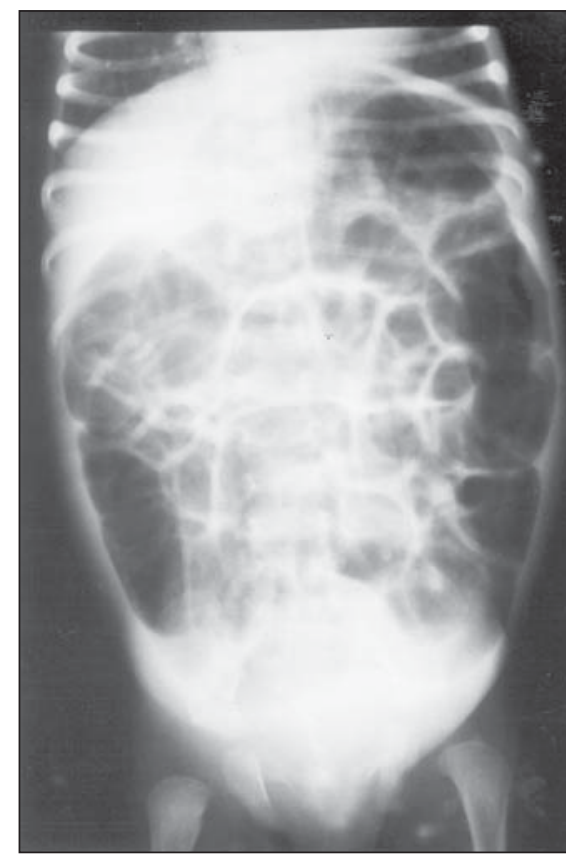

A

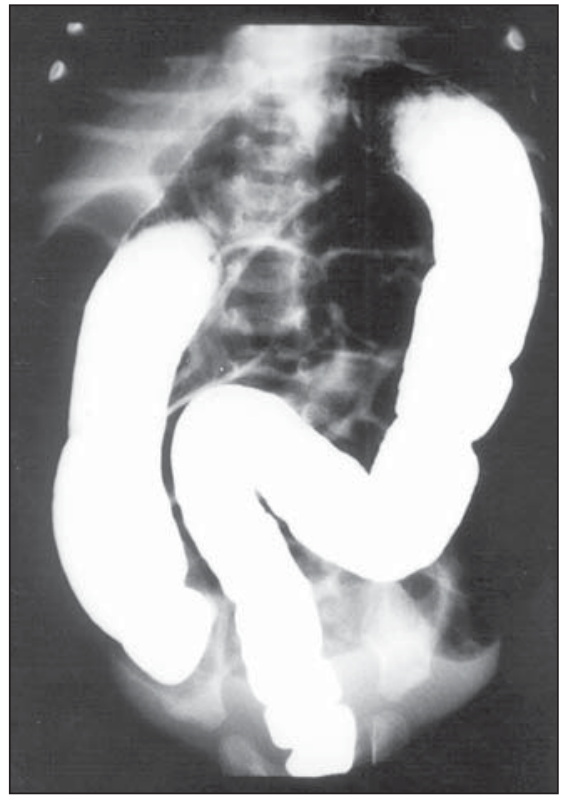

B

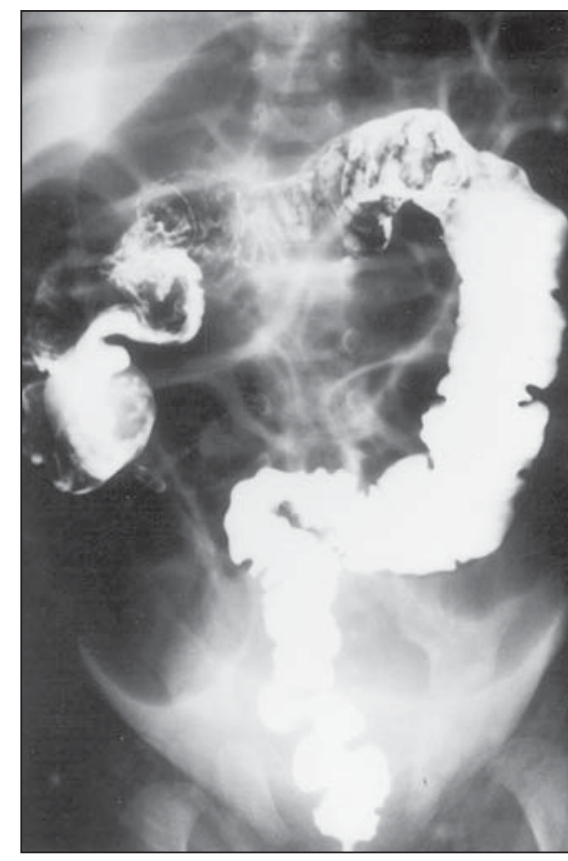

C

Figura 1. Caso 1. Radiografia do abdome (A) com distensão intestinal, sem ar no reto. 0 enema opaco (B) mostra encurtamento do cólon e perda da redundância das flexuras. Há retenção do contraste em todo o cólon na radiografia retardada após 24 horas (C).

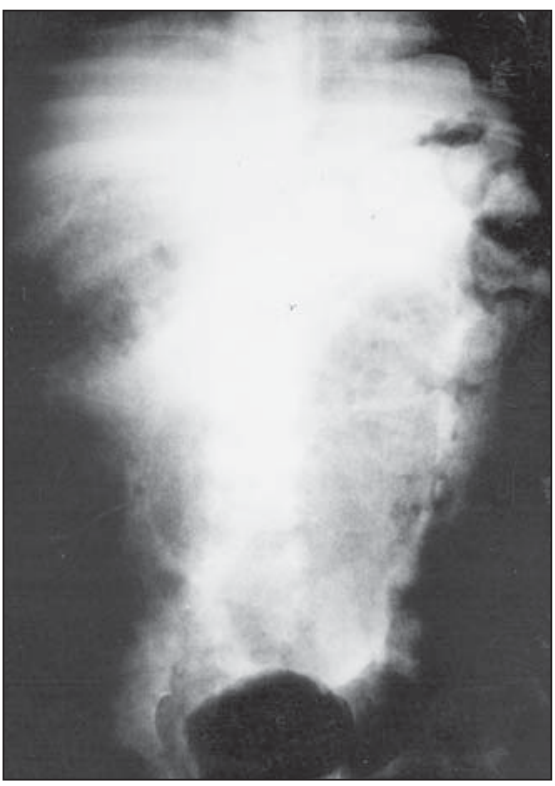

A

Figura 2. Caso 2. Grande conteúdo fecal na radiografia simples (A). 0 enema opaco (B) demonstra distensão do hemicólon direito e conteúdo fecal em meio ao contraste.

condição autossômica recessiva e poderia resultar de múltiplos eventos isquêmicos intra-uterinos ${ }^{(7)}$.

O diagnóstico precoce da doença de Hirschsprung é importante pela possibilidade de complicações sérias como enterocolite necrotizante e perfuração com peritonite. Quando todo o cólon é comprome-

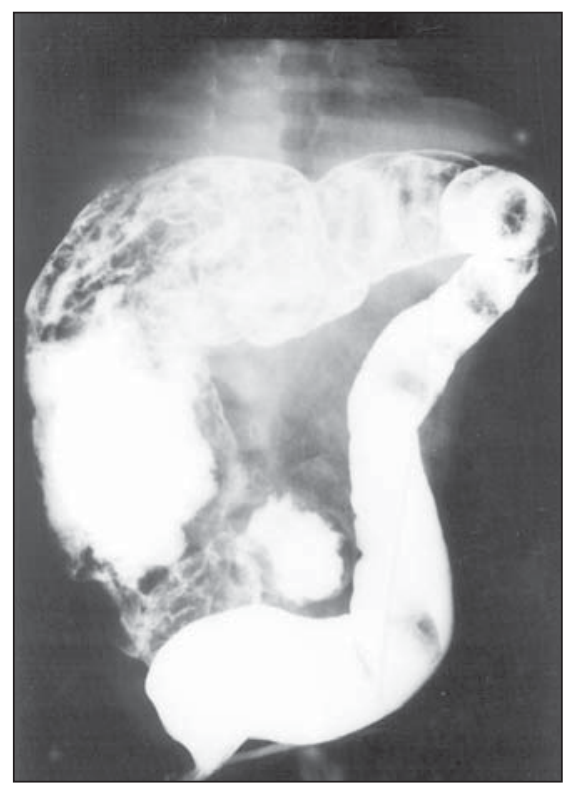

B

tido, o diagnóstico é geralmente tardio ou não realizado, resultando em maior taxa de mortalidade e complicações ${ }^{(6)}$.

A ATC representa cerca de 7,9\% de todos os tipos de doença de Hirschsprung e a ocorrência familial é maior $(20 \%)$ do que na forma clássica segmentar ${ }^{(3)}$. Também a enterocolite tem ocorrência maior
(25\%) na $\mathrm{ATC}^{(3)}$. Por estes motivos, a possibilidade diagnóstica de ATC deve ser sempre considerada diante de criança com evidências de obstrução intestinal.

Qualquer recém-nascido com sinais de obstrução intestinal baixa deve ser examinado com enema opaco com contraste hidrossolúvel. As causas de obstrução intestinal baixa nesta fase incluem: ânus imperfurado, síndrome da rolha meconial, íleo meconial, síndrome do hemicólon esquerdo curto, doença de Hirschsprung, síndrome da megabexiga, microcólon e hipoperistalse intestinal, atresias ou estenoses colônica, ileal e jejunal distal ${ }^{(2)}$.

Não há sinais radiológicos patognomônicos da $\mathrm{ATC}^{(\mathbf{8})}$. Em dois dos nossos casos as radiografias simples mostraram abundante conteúdo fecal retido em crianças maiores, respectivamente de dois e 12 anos de idade, o que reflete as dificuldades para o diagnóstico desta forma particular de aganglionose. No caso 1, além dos sinais radiológicos de obstrução, houve grande retenção do contraste na radiografia após 24 horas, que se constitui em um sinal radiológico significativo para o estudo destes pacientes. Sane e Girdany ${ }^{(\mathbf{9})}$ encontraram os seguintes aspectos radiológicos em uma revisão dos exames contrastados de 76 pacientes: microcólon (38\%), enema opa- 


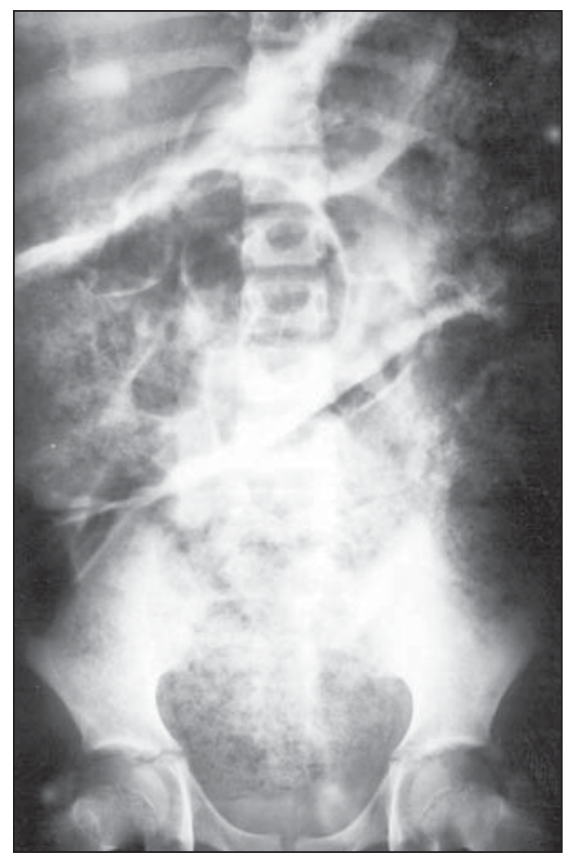

A

Figura 3. Caso 3. Radiografia do abdome (A) com distensão de alças intestinais e fecaloma. No enema opaco (B) há distensão de alças intestinais e abundante retenção de material fecal.

co normal $(21 \%)$, retenção prolongada do contraste $(21 \%)$, cólon curto e de calibre normal (19\%), presença de zona de transição $(15 \%)$, rolhas de mecônio $(8 \%)$, refluxo de contraste para o intestino delgado $(5 \%)$, contrações intestinais anormais (4\%) e megacólon (2\%). De Campo et al. ${ }^{(8)}$ confirmaram a ausência de achados radio-

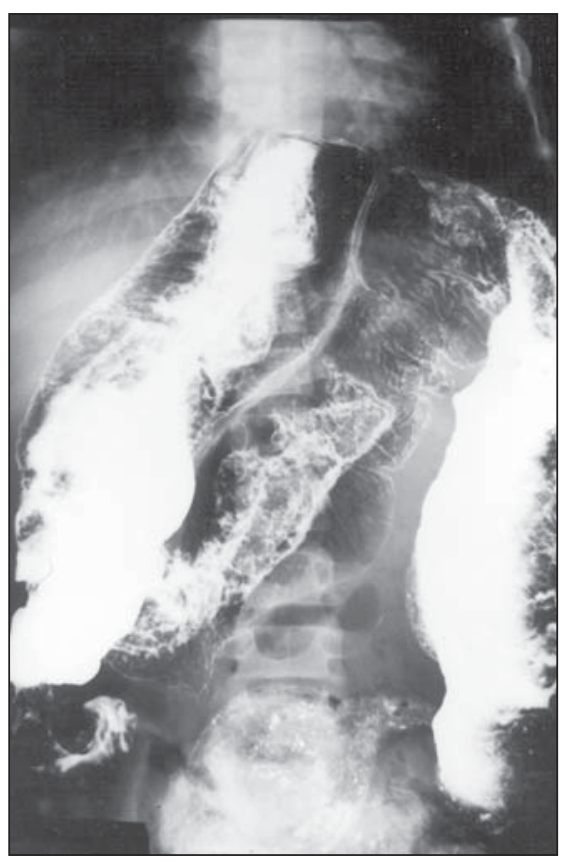

Sinais radiológicos de obstrução baixa de intestino delgado associados a história de doença de Hirschsprung em outro membro da família devem colocar como forte suspeita o diagnóstico de ATC, mas a confirmação definitiva somente será obtida com o estudo histopatológico e histoquímico. Portanto, a ATC deve ser sempre considerada em todo recém-nascido ou mesmo criança maior com evidências radiológicas de obstrução intestinal, quaisquer que sejam os achados de enema opaco.

\section{REFERÊNCIAS}

1. Soltero-Harrington LR, Garcia-Rinaldi R, Able LW. Total aganglionosis of the colon: recognition and management. J Pediatr Surg 1969;4:330-8.

2. Das Narla L, Hingsbergen EA. Case 22: total colonic aganglionosis - long-segment Hirschsprung disease. Radiology 2000;215:391-4.

3. Galifer RB, Frapier JM, Bosc O, et al. Forme colique totale de la maladie de Hirschsprung. À propos de 2 cas. Chir Pédiatr 1987;28:137-44.

4. Ikeda K, Goto S. Total colonic aganglionosis with or without small bowel involvement: an analysis of 137 patients. J Pediatr Surg 1986;21:319-22.

5. Talwalker VC. Aganglionosis of the entire bowel. J Pediatr Surg 1976;11:213-6.

6. Asch MJ, Weitzman JJ, Hays DM, Brennan LP. Total colon aganglionosis: report of nine cases. Arch Surg 1972;105:74-8.

7. MacKinnon AE, Cohen SJ. Total intestinal aganglionosis. An autosomal recessive condition? Arch Dis Child 1977;52:898-9.

8. De Campo JF, Mayne V, Boldt DW, De Campo M. Radiological findings in total aganglionosis coli. Pediatr Radiol 1984;14:205-9.

9. Sane SM, Girdany BR. Total aganglionosis coli. Clinical and roentgenographic manifestations. Radiology 1973;107:397-404. 